\title{
Design and Simulation of Microstrip Stub Low-pass Filter Based on ADS
}

\author{
Zhu Qiyuan \\ School of Information Science and Technology \\ Zhanjiang Normal University \\ Zhanjiang, China \\ Zhuqy1979@126.com
}

\author{
Huang Xiaotao \\ School of Information Science and Technology \\ Zhanjiang Normal University \\ Zhanjiang, China \\ Huangxt1980@126.com
}

\begin{abstract}
Richards transformation and Kuroda rule are briefly introduced firstly as the theoretical support of the research. Then, based on the Richards transformation and the Kuroda rule description, the structure of a microstrip low-pass filter and its designing principle are empirically analyzed through circuit simulation and layout simulation. Based on the ADS2011 software platform, we give an example to carry out the design and simulation by calling macro function of ADS. The simulation process contains four steps: Firstly, the basic values of the filter prototype are determined. Secondly, simulaton circuit is designed by using the Richards transformation and the Kuroda rule. Thirdly, intelligent computing for the microstrip line is carried out on the ADS2011 platform so as to form the layout. And fourthly, we employ the typing technique to modify the layout to control and ensure the exactness of the simulation. The simulation on the ADS software platform avoids the massive and complicated manual calculation and effectively shortens the designing period. Moreover, the satisfactory performance index is obtained
\end{abstract}

Key words - microstrip; low-pass filter; layout simulation; Richards transformation; Kuroad rule.

\section{INTRODUCTION}

To achieve the predetermined frequency characteristics, a filter which is a two port network is usually used in the system design. When the frequency is not high, the filter can be formed by components with lumped inductance and lumped capacitance. However, when the frequency is higher than $500 \mathrm{MHz}$ and the parasitic influence on the circuit cannot be ignored [1], the design of a filter system becomes a complicated work. Filters can be divided into low-pass filter, high-pass filter, band-stop filter and bandpass filter. The output of an ideal filter equals its input within the passband and zero within the stopband.

The low-pass filter with lumped parameter components can be transformed into a parameter distributed low-pass filter. The latter is realized through microstrip stub implementation, in which the Richard transform is used to replace the lumped components with the transmission line, and Kuroda rules is used to separate the filter components.

The structure of our paper is so arranged. The theory of Richards Transform and Kuroda rules are shown in Section II and Section III. Simulated and measured results are given in Section IV. At last the conclusions are presented in Section V.

\section{THE PRINCIPLE OF RICHARDS TRANSFORM}

Inductance and capacitance of the lumped components are turned into a section of equivalent transmission line, which is short circuited and open in terminal respectively. The input impedance of the transmission line, either it is short circuited or is open in the terminal, possess net reactance characteristic. This characteristic of the transmission line can be used to realize the transformation from lumped components to the distributed parameter ones. When a section of open (short) circuit transmission line is equivalent to the distributed inductance (capacitance) element, the input net-resistance impedance of a section of transmission line which is short circuited in the terminal and has characteristic impedance $\mathrm{Z}_{0}$ is

$$
Z_{\text {in }}=j Z_{0} \tan (\beta l)=j Z_{0} \tan (\theta),
$$

in which $\theta$ is the electric length. The expression of $\theta$ is

$$
\theta=\beta \frac{\lambda_{0}}{8}=\frac{2 \pi f}{v_{p}} \frac{v_{p}}{8 f_{0}}=\frac{\pi}{4} \frac{f}{f_{0}}=\frac{\pi}{4} \Omega,
$$


where $\lambda_{0} / 8$ is the length of the transmission line, $f_{0}=v_{p} / \lambda_{0}$ is work frequency, and $\beta, v_{p}, \Omega$ are respectively transmission constant, phase velocity, normalized frequency [2-3]. Substituting Eq. (2) into Eq. (1), we can find that the inductive lumped parameters element can be expressed as a short circuited transmission line, i.e.

$$
j X_{L}=j \omega L=j Z_{0} \tan \left(\frac{\pi}{4} \frac{f}{f_{0}}\right)=j Z_{0} \tan \left(\frac{\pi}{4} \Omega\right)=S Z_{0},
$$

where $S=j \tan \left(\frac{\pi}{4} \Omega\right)$ is exactly Richards transformation.

Similarly, the capacitive lumped element can also be replaced with a section of transmission line:

$$
j B_{C}=j \omega C=j Y_{0} \tan \left(\frac{\pi}{4} \Omega\right)=S Y_{0},
$$

which can be accomplished by the way as follows. According to Richards transformation, the lumped inductance is replaced with a section of short circuited transmission line, and the Lumped capacitance is replaced with a section of open transmission line.

\section{THE RULES OF KURODA}

The use of the additional transmission line can make the filter more easily realized in engineering [5]. Therefore, Kuroda proposes four sort of circuit transformation forms, as shown in Table I, where the inductance and capacitance represent respectively the short-circuit and open-circuit stub.

Table I . FOUR KURODA RULES

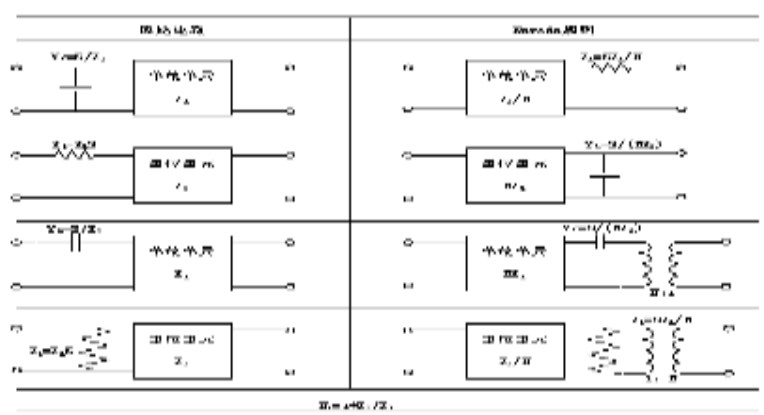

\section{DESIGN EXAMPLES OF MICROSTRIP STUB FILTER}

A new and practical microstrip stub low-pass filter is designed according the below indexes: cut off frequency is $f_{0}=4 G H z$; pass band ripple is 3 , of which attenuation out of band at two times the cutoff frequency is not less than $40 \mathrm{~dB}$; input impedance is $50 \mathrm{ohms}$; circuit board thickness $H=1 \mathrm{~mm}$ and relative dielectric constant is $\varepsilon_{r}=2.3$ For this we design a low-pass filter circuit with lumped parameters, and then select and use $3 \mathrm{~dB}$ ripple Chebyshev circuit, lastly utilize the ADS software to simulate a threeorder filter circuit with out of band at the cutoff frequency $F_{s}=4 G H z$ is larger than $40 \mathrm{~dB}$.

\section{A. Basic value}

For the three-order filter and Chebyshev circuit of the $3 \mathrm{~dB}$ ripples inside band, the component values of the lowpass filter prototype are [6]

$$
\begin{aligned}
& g_{1}=3.3487=L_{1} \\
& g_{2}=0.7117=C_{2} \\
& g 3=3.3487=L_{3}
\end{aligned}
$$

\section{B. The circuit transformation}

According to the above theory, the circuit can be transformed through Richards transform and Kuroda rules. In other words, we can use a section of transmission line of characteristic impedance $z_{0}=L$ replace lumped inductance or an open circuit output line of characteristic impedance $z_{0}=1 / C$ replace it. The transformation can be finished by calling macro function in $\mathrm{ADS}$, so that the complicated calculation process is avoided, and also the convenience, celerity and high efficiency of the software design are shown [7]. However, in actual engineering practice and the realization process of the equivalent series inductive impedance, the use of short circuited transmission line is more difficult than that of open circuited transmission line, so series and short circuited transmission line is usually changed into parallel and open transmission lines by using Kuroda rules, as shown in Fig. 1. 


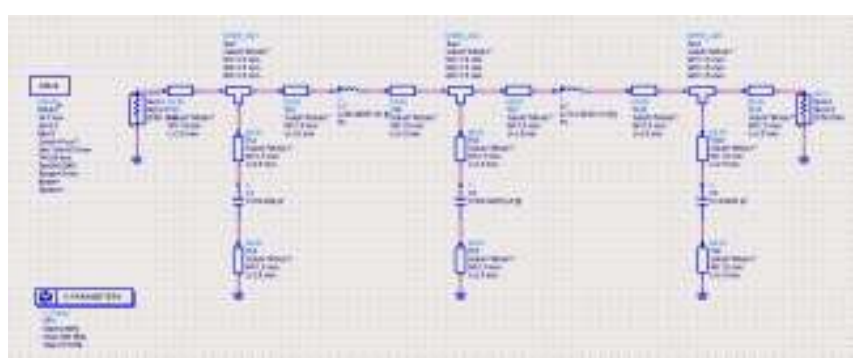

Figure 1. Microstrip Stub Low-pass Filter Schematic

\section{Intelligent Computing}

Using the ADS tool to complete the calculation of microstrip line, as shown in Fig. 2.

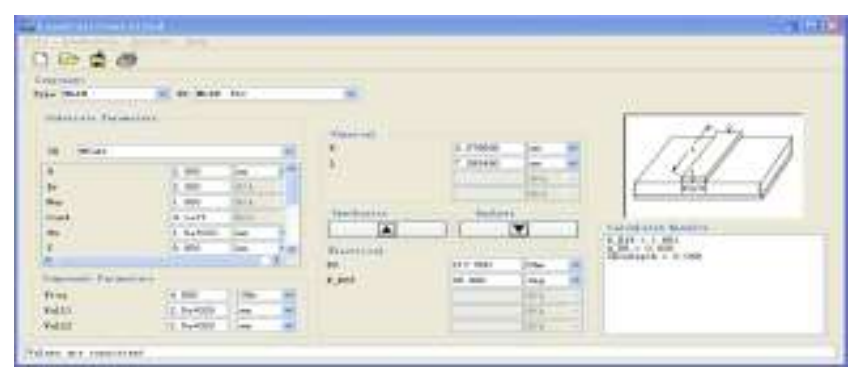

Figure 2. Calculation Window Size Microstrip Line

Through the calculation, we obtain the size data of a microstrip stub low-pass filter. The width and length of the microstrip are shown in table II .

Table II . THE CALCULATION OF MICROSTRIP STUB SIZE

\begin{tabular}{c|c|c|c|c}
\hline Knot & $\begin{array}{c}\text { Characteristic } \\
\text { impedance }\end{array}$ & $\begin{array}{c}\text { Phase } \\
\text { shift }\end{array}$ & W(Width) & L(Length) \\
\hline $\begin{array}{c}\text { Unit } \\
\text { components }\end{array}$ & $217.5 \Omega$ & $45^{\circ}$ & $0.0796 \mathrm{~mm}$ & $7.268 \mathrm{~mm}$ \\
\hline Stub1 & $64.9 \Omega$ & $45^{\circ}$ & $3.920 \mathrm{~mm}$ & $6.759 \mathrm{~mm}$ \\
\hline Stub2 & $70.3 \Omega$ & $45^{\circ}$ & $3.405 \mathrm{~mm}$ & $6.790 \mathrm{~mm}$ \\
\hline Stub3 & $64.9 \Omega$ & $45^{\circ}$ & $3.920 \mathrm{~mm}$ & $6.759 \mathrm{~mm}$ \\
\hline $\begin{array}{c}\text { system } \\
\text { impedance }\end{array}$ & $50 \Omega$ & & $5.974 \mathrm{~mm}$ & \\
\hline
\end{tabular}

\section{Generation and simulation circuit}

We use ADS simulation software to change transmission line into microstrip structure with the thickness $H=2 \mathrm{~mm}$ and the relative dielectric constant of the media $\varepsilon_{r}=2.3$ and increases the $\mathrm{T}$ joint in order to reduce the discontinuity. The modified circuit is shown Fig. 3.

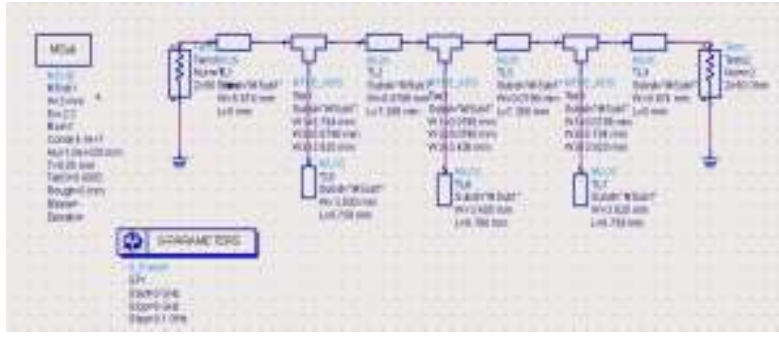

Figure 3. With the Terminal Load Stub Microstrip Low-pass Filter Schematic

The simulation results are shown in Fig. 4. From the simulation results Fig. 4 we can see that the effect is relatively good. However, when we use the software to generate layout (as shown in Fig. 5) and then simulation again, it can be seen that the simulation result is not very ideal. The main cause is that the method of layout simulation is different from that of the principle diagram. In order to achieve the ideal simulation results, we attempt to make changes to individual part of the layout and make the tuning of the transmission line [810], and then simulate. It is obvious that the effect improves notable and the results are close to the design indexes, which is shown in Fig. 6.

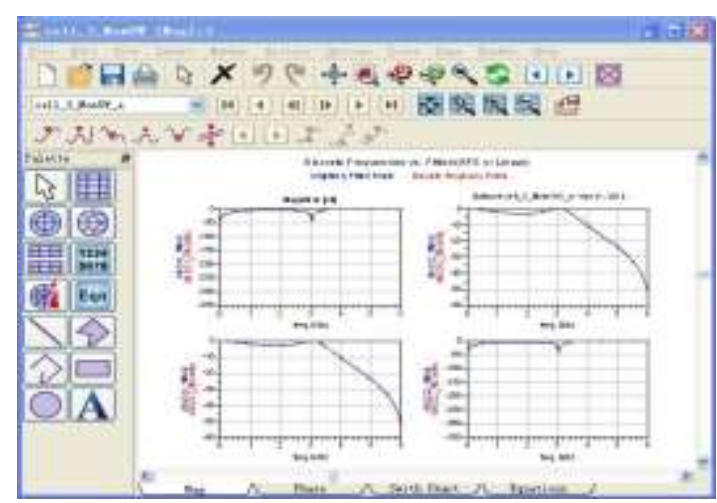

Figure 4. Microstrip Stub Filter Simulation Curve

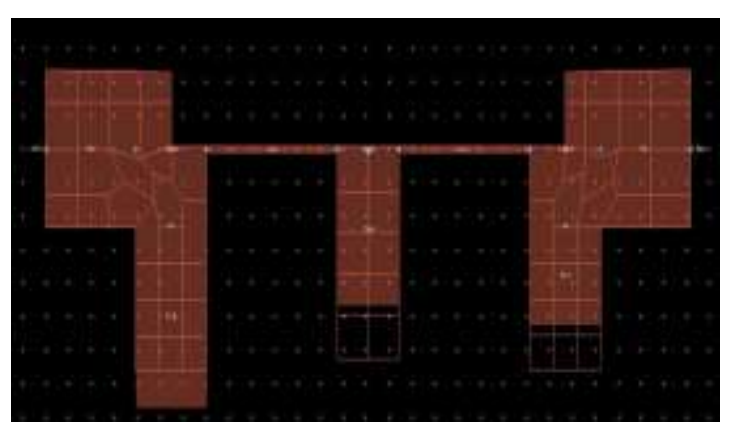

Figure 5. The layout of Microstrip Stub Low-passFilter Principle Diagram 


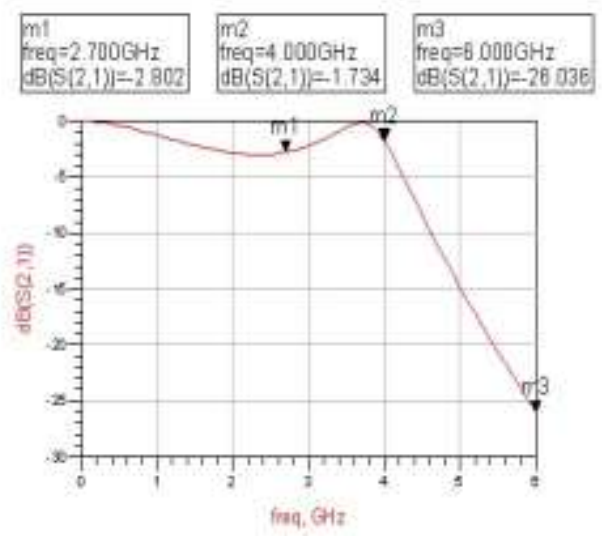

Figure 6. simulation data of meeting the technical index of the stub microstrip low-pass filter layout

The simulation results achieve the ideal performance after the modification. The filter circuit possesses a lot of characteristics such as compact structure, simple design, small size and come true easily, etc. Therefore both the design and the implementation of the microstrip stub lowpass filter based on Richards transform and Kuroads rules are feasible and it is worthy to be popularized in the microwave filter design and application.

\section{CONCLUSION}

In the design process of ADS microstrip stub lowpass filter, the reasonable and effective application of the Richards transform and Kuroda rules greatly reduces the complex computation process of the artificial and shorten the design cycle. The superiority of design software is showed. To both circuit simulation and layout simulation, the results can ultimately attain the requirement of performance indexes through tuning continuously in the adjustable range.
[1] Reinhold Ludwing,Pavel Bretchko . Design theory and application of RF circuit $[\mathrm{M}]$. beijing: Beijing Publishing House of electronics industry,2002.

[2] Li Zhengfan,Mao Fajun . Microwave and high speed circuit theory [M] . Shanghai: Shanghai Jiao Tong University press,2001,8:115127.

[3] Yang Jinwei . Design of RF filter based on Richards transform and Kuroads rules[J], Journal of Taizhou University 2006,28(3): 41-46 (in Chinese).

[4] M.-J. Park, "Dual-band, unequal length branch-line coupler with center-tapped stubs,"IEEE Microw. Wireless Compon. Lett., vol. 19,no. 10, Oct. 2009.

[5] Wei, C.L.; Jia, B.F.; Zhu, Z.J.; Tang, M.C.; , "Hexagonal dualmode filter with four transmission zeros," Electronics Letters, vol.47, no.3, pp.195-196, February 32011.

[6] Y. Wu, Y. Liu, and Q. Xue, "An analytical approach for a novel coupled-line dual-band Wilkinson power divider,"IEEE Trans. Microw.Theory Tech., vol. 59, no. 2, pp. 286-294, Feb. 2011.

[7] S. Gupta, A. Parsa, E. Perret, R. V. Snyder, R. J. Wenzel, and C. Caloz,"Group-delay engineered noncommensurate transmission line all-passnetwork for analog signal processing,"IEEE Trans. Microw. TheoryTech., vol. 58, no. 9, pp. 2392-2407, Sep. 2010.

[8] C.-F. Chen, T.-M. Shen, T.-Y. Huang, and R.-B. Wu, "Design ofmultimode net-type resonators and their applications to filter andmultiplexers,"IEEE Trans. Microw. Theory Tech., vol. 59, no. 4, pp.848-856, Apr. 2011.Article in a conference proceedings.

[9] F. Wei, L. Chen, X.-W. Shi, Q.-L. Huang, and X.-H. Wang, "Compactlowpass filter with wide stop-band using coupled-line hairpin unit,"IETElectron. Lett., vol. 46, no. 1, pp. 88-90, Jan. 2010.

[10] L. Li, Z.-F. Li, and J.-F. Mao, "Compact lowpass filters with sharpand expanded stopband using stepped impedance hairpin units,'IEEEMicrow. Wireless Compon. Lett., vol. 20, no. 6, pp. 310-312, Jun. 2010.

\section{REFERENCES}

